\title{
Three-year caregiver's reports on driving performance: A divination or diagnostic tool for fitness to drive in Parkinson Disease patients
}

\author{
Hoe C. Lee \\ School of Occupational Therapy and Social Work, Curtin Health Innovation Research Institute, Curtin University, Bentley, Australia; \\ h.lee@curtin.edu.au \\ Received 23 November 2012; revised 28 December 2012; accepted 7 January 2013 \\ Copyright (C) 2013 Hoe C. Lee. This is an open access article distributed under the Creative Commons Attribution License, which \\ permits unrestricted use, distribution, and reproduction in any medium, provided the original work is properly cited.
}

\begin{abstract}
Objectives: This study aims to examine whether information provided by spouse or relatives can be employed to identify PD patients with deteriorated driving performance, using three-year caregiver's reports on their driving ability as the outcome measure. Methods: Fifty-three idiopathic PD subjects were assessed on open roads. Prior to the driving assessment, participants were examined by a geriatrician with various clinical assessments. The caregivers filled out a questionnaire, the scores of which is a reflection of their concern on driving performance of their PD relatives. The same measurements were collected for the subsequent two years. Hierarchical Poisson regression analysis, adjusting for gender, age and driving exposure (hours of driving per week), was then undertaken to determine whether the measures of driving assessment were associated with the score of the questionnaire. Results: During the three-year period, all PD participants were rated at least 3 questions positive in the caregiver's questionnaire; the worst participant was rated positive eight times. Except the assessment criteria to gauge the traffic rules and regulations compliance, all other measures of the driving assessment were found to be significantly associated with the information provided by the caregivers. Conclusions: This study demonstrated that the information provided by caregivers was useful to identify PD patients with deteriorated driving performance. If adopted as part of the off-road driving assessment for PD patients, the questionnaire can provide reliable information to clinicians.
\end{abstract}

Keywords: Parkinson Disease Drivers; Caregiver; Driving Assessment; On-Road Screening; Drivers; Road Safety

\section{BACKGROUND}

People suffering from Parkinson Disease generally stop driving at the age of 68; eight years earlier than the general population, and the condition is the sixth highest cause of disease-related driving cessation in Australia [1]. Driving requires numerous skills to be learnt, coordinated and continuously adapted in various traffic scenarios with time-based pressures [2]; minor mistakes in driving may result in severe or fatal crashes [3]. Symptoms in $\mathrm{PD}$, such as motor tremors, bradykinesia, postural instability, rigidity and generalized slowness are known to affect driving ability of PD drivers in negotiating roundabouts, turning across traffic, travelling at high speeds and busy urban environments $[4,5]$.

Through driving, individuals are able to maintain independence, access to the community and preserve their self-efficacy, therefore longer licence retention can be very beneficial to good quality of life [6]. Nowadays the increasing complexity of road systems places additional demands on the cognitive, physical and sensory systems on road users [7]. Drivers with PD face further challenges as the pathological deficits as well as the side effects of medications can interfere with driving performance [8]. Nevertheless it is unethical to cancel licences based upon diagnosis of PD alone [9], as many drivers with PD are able to overcome barriers using their extensive driving experience and knowledge of road systems or they can compensate for the declining ability [10]. Most of them also self-monitor and self-regulate their driving habits by avoiding potentially difficult or risky situations, such as not driving on the freeway, avoiding peak congestion periods or having a co-pilot [5]. 
A road test is the gold standard for driving assessment, however, in most countries there are no standards for how the assessment procedures should be conducted [11]. The on-road assessment is costly and time-consuming [12] and the need for drivers with PD to undergo annual driving reviews may contribute to the early cessation of driving [1]. General practitioners are often required to determine the driving ability of their patients, even though majority of them have not been trained or actually observed a driving assessment [13]. Their decision of determining the cancellation of a licence is mostly made by medical expert opinions and/or based on subjective judgment of the results of the psychometric assessments conducted in the clinic [14].

A previous study reported that clinical assessment tools, such as the Timed Up and Go (measures ability to stand up, walk for 3 metres and return to the chair), Unified Parkinson's Scale and Mini Mental Status Examination (MMSE) cannot predict the driving performance of the PD patients, but the relatives can provide valuable information in the making decision [8]. The authors recommended the development of a tool, through which spouses or relatives can methodologically collect and record the driving behaviours of PD patient over a period of time. The information can improve the validity and reliability of the licence procedures. Using three-year records of a questionnaire assessing driving ability as outcome measure, this study aims to examine whether information provided by caregivers can be employed to identify PD patients with deteriorated driving performance.

\section{METHODS}

\subsection{Methodology}

This longitudinal study over a period of three years targeted 53 PD patients who were living in the community, not having incurred five or more demerit points in the past two years and driving at least four hours each week. Participants with PD had a confirmed diagnosis with no history or evidence of additional neurological impairment that may affect driving and were recruited from local specialist PD clinics and neurologists. All subjects were screened for acute, confounding medical and psychiatric conditions. Volunteers were excluded either if they scored less than 26 on the Mini Mental State Examination (MMSE) or their visual acuity worse than 6/12 corrected on Snellen chart testing.

\subsection{Data Collection Procedure}

The study was approved by the Human Research Ethics Committee of the researchers' institution. During October 2009, PD drivers between 60 to 80 years old residing in Perth, Western Australia were invited to take part in the study voluntarily. Each assessment included a 30-minute initial interview to collect information surrounding driving habits and a 45-minute on-road driving session. In the event that the performance of a participant indicated unsafe driving practice as judged by the principal investigator, free counselling and advice were available. With the permission of the individual concerned, a follow-up referral would be arranged to address the issue.

Prior to the driving assessment, the PD participants were examined by a geriatrician to ensure all driving and clinical tests were undertaken when the participants were optimally treated. Standard clinical measures for PD patients including the overall UPDRS, motor UPDRS, H-Y score, levodopa equivalents, number of doses per day, MMSE, IQ code for dementia, Epworth Sleepiness Score, and Timed Up and Go Test were administered to all participants.

\subsection{Caregiver's Perceptions of PD Participant's Driving Ability and State Record of Traffic Violation}

The caregiver or relative was asked to fill out questionnaires on their perception of the PD participant's driving ability. The Carer Questionnaire with 11 quetions emphasised the difficulties and safety of PD patients in driving (refer to Table 1). The scores of the questionaire are a reflection of caregiver's concern on the driving performance of their spouse or relatives. Previous study reported that the questionnaire can provide valuable information on PD driver's ability: 55\% variability in the driving performance scores of the PD drivers can be explained by the questionnaire [8]. Written authorization to access official driving records of traffic violations was also sought from all participants. Immediately after the initial screening, driver violation points of participant were retrieved. Anonymity and confidentiality on the information provided was assured. Caregiver's perception on driving ability of PD participants and driver violation points in subsequent two years, 2010 and 2011, were collected.

\subsection{Driving Performance Assessment}

All personnel involved in the driving assessment were unaware of the medical conditions of the participants. Driving performance was assessed in the participant's car along a $15 \mathrm{~km}$ route chosen by the participants in his/her neighborhood to cover a range of typical driving situations, such as roundabouts, traffic lights, pedestrian crossings and T-junctions. An accredited professional driving instructor, experienced in assessment of disabled drivers, sat in the front passenger seat and was responsible for maintaining vehicle safety. A driver trained occupa- 
tional therapist, experienced in driving assessment and rehabilitation, sat in the rear seat. Both assessed driving ability of participants using well defined on-road assessment criteria (Refer to Table 2). The measures of driving tasks were designed with reference to the three major levels of driving behaviours in the Michon model of car driving [15]. Strategic level behaviors often occur before driving such as planning the shortest route to the destination. Tactical level behaviours happen while the driver responds to regulatory traffic signs. Operational level behaviors include second-to-second driving manoeuvres in adjustment of car position to maintain reasonable distance from the car in front. A higher score of the measures reflects better driving performance.

\subsection{Statistical Analysis}

All data was coded and analyzed using STATA version 10 [16]. Reliability of the driving task measure was assessed by Cronbach alpha coefficient. The repeated measure of the carer's information was the main outcome variable of interest. Hierarchical Poisson regression analysis based on generalized estimating equations [17] was undertaken to determine a subset of the driving tasks affecting the number of score of the Caregiver's questionnaires over the three-year period. The method is an extension of standard Poisson regression to handle the hierarchical data (repeated outcomes from the same subject). It accommodates the inherent correlation of the repeated observations clustered within a subject and provides robust standard errors for the Poisson regression coefficients, so that correct inferences can be made. A statistically significant relationship would provide evidence of validity of the caregiver's information to identify PD patients with deteriorations in particular area of their driving performance.

\section{RESULTS}

\subsection{Descriptive Analysis}

The age of the $53 \mathrm{PD}$ participants ranged between 61 and 70 years (mean 69.3, S.D. 8.3), and 22\% of the drivers were female. H-Y score [Mean (SD): 1.6 (0.5)]; UPDRS [Mean (SD): 7.8 (1.1)]; MMSE [Mean (SD): 29 (1.2)], IQ code for dementia [Mean (SD): 3.2 (0.4)]; and Timed Up and Go Test [Mean (SD): 9.3 (2.2)] were recorded. The self-reported estimated driving hours per week for each individual did not vary significantly throughout the three-year period (overall mean 9.7; S.D. 5.5), according to repeated measures ANOVA (p-value $=0.623$ ). Shopping and attending medical appointments were the most common reasons that participants drove. Around 15\% of them were professional drivers prior to retirement, which required driving a vehicle regularly. None of the participants requested or was required a follow-up referral or counselling.

All participants were rated "Yes" at least in three questions of the carer's questionnaire within the past three years by the carers, while the maximum "positive" count was eight. The most common question rated positive by the caregiver's was "Do you think the patient has difficulty in turning the steering wheel, changing gears, pressing the pedals?", whereas, the least common was "Has the patient received any speeding tickets or traffic violtions?” According to the state records, all participants incurred at least one driver violation point within the past three years, while the maximum yearly point count was three. The most common reason of traffic violation reported by the participants was illegal parking.

\subsection{Reliability Check}

The measurement properties of the 7 driving tasks assessed during road test were next examined. The Cronbach alpha coefficient was found to be 0.829 , confirming the internal consistency of the scales that measured performance in the driving tasks. There was no significant correlation between each measure and the psychometric assessments (overall UPDRS, motor UPDRS, H-Y score, levodopa equivalents, number of doses per day, MMSE, IQ code for dementia, Epworth Sleepiness Score, and Timed Up and Go Test). However, as expected there was substantial negative correlation between each measure and the age of participants (correlation ranged from 0.457 to 0.612 ), suggesting that driving performance of the current PD cohort could deteriorate with increasing age.

\subsection{Inferential Analysis}

The objective was to identify a subset of driving tasks related to the total score of the Caregiver's questionnaires. Two of the 53 participants could not be contacted at second and/or third year, resulting in $\mathrm{N}=150$ observations and a loss-to-follow up rate of $6.9 \%$. Results of the hierarchical Poisson regression analysis of the caregiver's questionnaire are presented in Table 3. Adjusting for age, gender and individual driving exposure, except Traffic Sign Compliance and Traffic Light which were designed to measure strategic level of the PD participants, all other measures of the driving tasks were found to be significantly associated with the caregiver's questionaire score. Goodness of fit of the hierarchical Poisson regression model was satisfactory (Chi-square = 157.19, p-value $=0.712$ ). Hierarchical regression analysis of the state record of violation points produced insignificant result and was omitted from the reported results for brevity. The agreement between the scores of the Cargiver's questionnaires and state record of traffic violation of the PD participants was not significant (Kappa statis- 
Table 1. The Carer Questionnaire on perception of participant’s driving ability.

\begin{tabular}{|c|c|}
\hline Items & Response \\
\hline 1. Do you feel uncomfortable in any way driving with the patient? & Yes/No \\
\hline 2. Have you noticed any abnormal or unsafe driving behaviour? & Yes/No \\
\hline 3. Has the patient had any crashes within the last 12 months? & Yes/No \\
\hline 4. Do you think the patient has difficulty in turning the steering wheel, changing gears, pressing the pedals? & Yes/No \\
\hline 5. Has the patient received any speeding tickets or traffic violations? & Yes/No \\
\hline 6. Are other drivers forced to drive defensively to accommodate the patient's errors in judgement? & Yes/No \\
\hline 7. Has the patient had near-misses that could be attributed to their Parkinson's disease? & Yes/No \\
\hline 8. Do you think the patient reacts too slowly to traffic situations? & Yes/No \\
\hline 9. Has the patient ever fallen asleep at the wheel since they were diagnosed with Parkinson's disease? & Yes/No \\
\hline 10. Have others commented on the patient's unsafe driving? & Yes/No \\
\hline 11. Do you think the patient is unsafe to drive? & Yes/No \\
\hline Total "Yes" score & \\
\hline
\end{tabular}

*Scoring procedures: 11 questions to be asked by medical practitioner; one point for each “yes” answer; maximum possible score for each questionnaire is 11.

Table 2. On-road driving performance of the PD participants.

Tasks required to perform by the participants

\section{Measure (Maximum possible score)}

Road Use Obligation: Driving manoeuvres in seven roundabouts ${ }^{\#}$

Traffic Sign Compliance: Manoeuvres through four pedestrian crossing, with a "5 mile/hr" speed control sign and a speed bump"

T-Junction: Manoeuvres before and through T-junctions ${ }^{@}$

General Driving Skill: The steering and breaking behaviour throughout the assessment ${ }^{@}$

Traffic Light: Manoeuvres before and through traffic lights ${ }^{*}$

Normal Driving: General driving behaviour throughout the experiment ${ }^{\# @ ~}$

Use of Indicator: Correct use of indicators throughout the assessment ${ }^{\#}$
Being certain of obligation, proceed when has opportunity to do so, give appropriate signals, give signal not too short or long, do not give wrong signal. (35)

Slow down in approaching the crossing, do not stop when it is not necessary, do not hit the speed bumps, give way to pedestrians crossing and comply with the 5 mile/hr rule. (20)

Do not approach intersection too slowly, do not brake or swerve at last minute, do not approach intersection too fast, look both ways in approaching intersection, do not proceed if way is not clear, proceed when has opportunity to, will give way (right hand rule), do not turn across oncoming traffic, do not swing too wide on corner, do not cut corner, do not swing too wide on corner and do not turn across oncoming traffic. (12)

Erratic movement of the steering wheel, do not put arm and elbow out of window, do not allow steering wheel to self centre, do not incorrectly position their hand, do not depress clutch before brake in high gear whilst stopping, do not stop too far from given mark, do not stop too suddenly, do not stop too slowly in quick stop test and do not stall engine. (50)

Do not stop in wrong position, do not stop when not necessary, do not start before signal turns green, do not proceed on amber when could have stopped safely, do not proceed on red signal, do not cross continuous white line and do not fail to notice lights. (7)

Keep to left, drive with reasonable speed, do not drive with erratic speed, do not take erratic course, use rear view mirror frequently, do not exceed speed limit, do not follow closely to the front car, do not overtake unnecessarily, do not veer over the centre line unless overtaking. (10)

One point for each correct use of indicators. (27)

Note: Measure to assess \# Operational level, ${ }^{@}$ Tactical level, *Strategic level, of the Michon model of car driving. 
Table 3. Hierarchical Poisson regression results for scores of the Carer's questionnaires, adjusting for clustering and individual driving exposure (hours per week), $\mathrm{N}=150$.

\begin{tabular}{cccccc}
\hline Variable & Mean (SD) & Incidence rate ratio & p-value & \multicolumn{2}{c}{ 95\% confidence interval } \\
\cline { 4 - 6 } & $22.8(2.13)$ & 1.06 & 0.003 & 0.83 & Upper \\
\hline Use of Indicator & $17.2(3.59)$ & 0.89 & 0.023 & 0.71 & 0.21 \\
Road Use Obligation & $6.43(3.52)$ & 0.88 & 0.029 & 0.69 & 1.17 \\
Normal Driving & $4.56(2.41)$ & 0.89 & 0.039 & 0.88 & 1.13 \\
T-Junction & $34.8(6.16)$ & 1.17 & 0.047 & 0.79 & 1.34 \\
General Driving Skill & $9.7(1.82)$ & 0.91 & 0.241 & 0.87 & 1.35 \\
Traffic Sign Compliance & $4.89(1.41)$ & 1.07 & 0.391 & 0.95 & 1.13 \\
Traffic Light & $69.3(8.3)$ & 0.96 & 0.524 & 0.92 & 1.14 \\
Age & $9.7(5.5)$ & 0.87 & 0.634 & 0.62 & 0.93 \\
Exposure (hr/week) & - & 0.79 & 0.415 & 0.73 & 1.12 \\
Gender & & & &
\end{tabular}

tic $=0.21$, p-value $=0.452$.

\section{DISCUSSION}

In this study, appropriate driving tasks were chosen to assess PD drivers in their neighbourhoods. Seven internally consistent measures of driving task were used to assess each participant's performance in these tasks. The negative correlation between individual measures of the driving tasks and age confirmed that driving skills in PD generally decline with age, however the clinical and psychometric measurements have no association with any measures of the driving performance of PD participants. A similar result was reported in another study that the driving performance of the PD participants could not be predicted by the commonly-used clinical assessments by general practitioners [8].

This three-year longitudinal study further investigated the association between the caregiver's information and driving performance of the PD participants. The hierarchical Poisson regression analysis showed that PD participants rated poorly by the caregivers did not perform well in the driving tasks that require high level of functioning in tactical and operational level [15]. The results are consistent with previous findings that the caregiver's questionnaire can provide reliable information on-road driving behaviors of PD drivers [8].

The violation points record of PD did not associate with either the performance of the PD participants or the score of the caregiver's questionnaire. One possibility is that deficits in particular functions of the PD drivers may lead to an impaired driving ability but does not extend to a higher likelihood of accumulation of violation points. The current study has identified the PD participants have deteriorated in particular aspects of driving. Due to the small sample size further links to the caregiver's questionnaire with PD drivers at high risk of road safety is impossible. Other limitations of the study included unequal gender distribution (78\% male). Therefore, gender was controlled for in the hierarchical Poisson regression analysis. The participants who volunteered for this study cannot be taken as representative of the population of older drivers, since the sample was notrandomly selected but only covered some sectors of the community. Selection bias was therefore unavoidable in the recruitment of the PD participants. However, random sampling was neither possible nor practical in this type of study. The majority of the participants were at $\mathrm{H}-\mathrm{Y}$ stage one or stage two, indicating their functional abilities were only minimally affected by the PD symptoms. The driving assessment was conducted during optimal on time medication and does not address the impact of symptom fluctuations in PD.

Our findings suggest that in making decisions on licence retainment or cancellation of PD patients, more weight should be given to the information provided by their caregivers. The use of the questionnaire can provide a more systematic ways to capture the driving performance of PD drivers over a period of time. The caregiver's questionnaire could be adopted as a screening tool to gain reliable information about driving performance of PD patients prior to administering a more detailed but expensive road test.

\section{ACKNOWLEDGEMENTS}

This study was supported by a research grant from Curtin University. The author is indebted to the staff of the Parkinson's disease Clinic, Osborne Park Hospital, Western Australia for assisting the recruitment and assessment of the PD participants.

\section{REFERENCES}

[1] Access Economics (2010) Federal policy initiatives: A new approach to Parkinson's disease. Parkinson's Australia, Melbourne.

[2] Evans, L. (2006) Traffic safety. 2nd Edition, Science Ser- 
ving Society, Michigan.

[3] Molnar, A., Marshall, H.L. and Man-Son-Hing, K. (2006) Clinical utility of office based cognitive predictors of fitness to drive in persons with dementia: A systematic review. Journal of American Geriatrics Society, 54, 18091824. doi:10.1111/j.1532-5415.2006.00967.x

[4] Lavezza, A. and Martin, S.L. (2006) The clinical evaluation of psychosocial factors. In: Pellerito, J.M., Ed., Driver Rehabilitation and Community Mobility, Elsevier Mosby St. Louis, 153-161.

[5] Amick, M.M., Grace, J. and Ott, B.R. (2007) Visual and cognitive predictors of driving safety in Parkinson's disease patients. Archives of Clinical Neuropsychology, 22, 957-967. doi:10.1016/j.acn.2007.07.004

[6] Siren, A., Hakamies-Blomqvist, L. and Lindeman, M. (2004) Driving cessation and health in older women. Journal of Applied Gerontology, 23, 58-69. doi:10.1177/0733464804263129

[7] Lee, H., Cameron, D. and Lee, A. (2003) Assessing the driving performance of older adult drivers: On-road versus simulated driving. Accident Analysis and Prevention, 35, 797-803. doi:10.1016/S0001-4575(02)00083-0

[8] Cordell, R., Lee, H.C., Granger, A., Vieira, B. and Lee, A. H. (2008) Driving assessment in Parkinson's disease: A novel predictor of performance. Movement Disorders, 23, 1217-1222. doi:10.1002/mds.21762

[9] Tröster, A.I. and Woods, S.P. (2007) Neuropsychological aspects. In: Pahwa, R. and Lyons, K.E., Eds., Handbook of Parkinson's Disease, 4th Edition, Informa Healthcare, New York, 109-130.

[10] Stolwyk, R.J., Triggs, T.J., Charlton, J.L., Lansek, R. and Bradshaw, J.L. (2005) Impact of internal versus external cueing on driving performance in people with Parkinson's disease. Movement Disorder, 20, 846-857. doi:10.1002/mds.20420

[11] Innes, C.R.H., Jones, R.D., Anderson, T.J., Hollobon, S.G. and Dalrymple-Alford, J.C. (2009) Performance in normal subjects on a novel battery of driving-related sensory-motor and cognitive tests. Behaviour Research Methods, 41, 284-894. doi:10.3758/BRM.41.2.284

[12] Bedard, M., Parkkari, M., Weaver, B., Riendeau, J. and Dahlquist, M. (2010) Assessment of driving performance using a simulator protocol: Validity and reproducibility. American Journal of Occupational Therapy, 64, 336-340. doi:10.5014/ajot.64.2.336

[13] Adler, G., Rottunda, S., Bauer, M. and Kuskowski, M. (2000) The older driver with Parkinson's Disease. Journal of Gerontological Social Work, 34, 39-49. doi:10.1300/J083v34n02 05

[14] National Road Transport Commission (2003) Assessing fitness to drive. 3rd Edition, Austroads Incorporated, Sydney.

[15] Michon, J.A. (1985) A critical review of driver behaviour models: What do we know, what should we do? In: Evans, L. and Schwing, R.C., Eds., Human Behaviour and Traffic Safety, Plenum, New York, 485-520. doi:10.1007/978-1-4613-2173-6 19

[16] Stata Corporation (2009) STATA release 10. Stata Corporation, College Station.

[17] Hardin, J.W. and Hilbe, J.M. (2002) Generalized estimating equations. CRC, Boca Raton. doi:10.1201/9781420035285 